\title{
Reduction of factor VII coagulant activity (VIIC), a risk factor for ischaemic heart disease, by fixed dose warfarin: a double blind crossover study
}

\author{
Leon Poller, Peter K MacCallum, Jean M Thomson, William Kerns
}

\begin{abstract}
An increase in factor VII coagulant activity is known to be an important risk factor for ischaemic heart disease. Four hundred and eight healthy male Post Office workers were screened in an occupational survey. Sixty eight (16.5\%) of these had values of factor VII coagulant activity $>1.0 \mathrm{SD}$ above the age related mean. A randomised doubleblind crossover study was undertaken to investigate the effect of a fixed daily minidose of warfarin $(1 \mathrm{mg})$ on the high activities of factor VII in these men. Forty two agreed to enter the study and 40 completed it. Their mean factor VII coagulant activity before warfarin treatment was $135 \cdot 9 \%$. Treatment with a fixed minidose of warfarin significantly reduced factor VII coagulant activity to $124.6 \%$; there was no change on placebo. The prothrombin time was also significantly prolonged on active treatment although all the results remained within the normal range.

These findings suggested a fixed minidose warfarin regime might be useful in the primary prevention of ischaemic heart disease by reducing high activities of factor VII.
\end{abstract}

High activities of the procoagulant clotting zymogen factor VIIC were shown to be an important risk factor for ischaemic heart disease and to be of even greater significance than cholesterol in the first five years after screening. ${ }^{12}$ Warfarin given in conventional adjusted doses according to the response of prothrombin time reduces the activity of factor VIIC. Bern et al, however, recently showed that much less intense treatment with a small fixed dose of warfarin ( $2 \mathrm{mg}$ daily) prevented blocking of central venous catheters in patients with malignant disease. ${ }^{3}$ Subsequently, we showed that an even smaller fixed dose $(1 \mathrm{mg}$ warfarin daily) significantly reduced the incidence of postoperative deep vein thrombosis. ${ }^{4}$

The possibility that $1 \mathrm{mg}$ warfarin daily might also reduce raised factor VII coagulant activity in healthy individuals was considered in the present study. We assessed the effects of this regimen in a randomised double blind crossover study in men with high factor VII coagulant activity.

\section{Patients and methods}

DESIGN OF STUDY

Four hundred and eight healthy male Post Office workers (age range 18-65 years) took part in an occupational screening programme during which their prothrombin time and factor VII coagulant activity were measured. Factor VII coagulant activity increases with age. ${ }^{56}$ Forty two of the 68 men with high activity ( $>1 \mathrm{SD}$ above the age related mean) agreed to participate by written informed consent.

In this selected group a repeat assay was performed during the three week period before the start to establish a pretreatment baseline. The 42 men were then allocated by random numbers to receive either warfarin $(1 \mathrm{mg} /$ day) or matched placebo for an initial twelve weeks. A further blood sample was then taken and the treatment regimens were crossed over for the second twelve weeks. The tests were then repeated.

\section{LABORATORY METHODS}

Fasting venous blood samples were taken between 0800 and $0900 \mathrm{~h}$ on each occasion by means of a 19 gauge butterfly and plastic syringes. The blood (9 volumes) was anticoagulated with $3 \cdot 2 \%$ trisodium citrate (1 volume) and centrifuged at $2000 \mathrm{~g}$ for 15 minutes. Factor VII coagulant activity and prothrombin times were estimated in duplicate within one hour of sample collection. All assays were performed blind.

\section{Factor VII coagulant activity assay}

This was estimated in a one stage method with factor VII-deficient beagle plasma. ${ }^{7}$ A standard curve was obtained from pooled plasma prepared from 20 blood donor volunteers. The participants' results were expressed as a percentage of the value from the normal pool. Inter assay variation was assessed by the International Standard for factor VII coagulant activity (National Institute for Biological Standards and Control, London). The same batches of reagents were used throughout the study.

\section{Prothrombin time}

Prothrombin time was measured with Manchester Reagent thromboplastin. Inter batch variation was excluded by the use of calibrated lyophilised normal reference plasma samples prepared at the UK Reference Laboratory for Anticoagulant Reagents and Control. 
Figure 1 Factor VII (2SE)) before and after 12 weeks on a fixed daily dose of $1 \mathrm{mg}$ warfarin. $p=0.03$ coagulant activity (mean

^Significant fall,

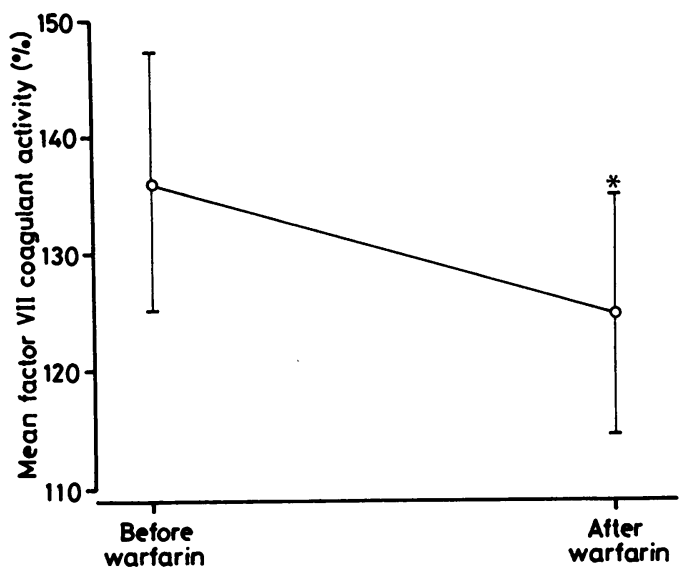

STATISTICAL ANALYSIS

We used log transformed values of factor VII coagulant activity because their distribution was positively skewed. Differences between and within groups were analysed by a repeated measures analysis of variance. ${ }^{8}$ We examined significant differences in detail using the Tukey range test ${ }^{8}$ as required. Analysis was performed on an "intention-to-treat" basis.

\section{Results}

Forty of the 42 men completed the study. Two withdrew for non-medical reasons. The age range was $31-61$ years (mean $47 \cdot 1$ ). Compliance, estimated by counting the returned tablets, was $90 \%$ throughout the study in both groups.

When the results of the two groups were combined, fixed minidose warfarin was found to be associated with a significant reduction in factor VII coagulant activity, with a mean fall of $11.3 \% \quad(p=0.03)$ from the values immediately before the start of warfarin treatment. There was a fall in mean factor VII coagulant activity from $135.9 \%$ before warfarin treatment $(95 \%$ confidence limits $124 \cdot 8-147 \cdot 0 \%)$ to $124 \cdot 6 \%(114 \cdot 4-134 \cdot 8 \%)$ after 12 weeks' warfarin treatment (fig 1 ).

Figure 2 shows the serial changes in factor

Figure 2 Factor VII coagulant activity (mean (2SE)) during treatment with a fixed daily dose of 1 mg warfarin and during treatment with placebo. * Significant difference after warfarin treatment, $p<0.05$.

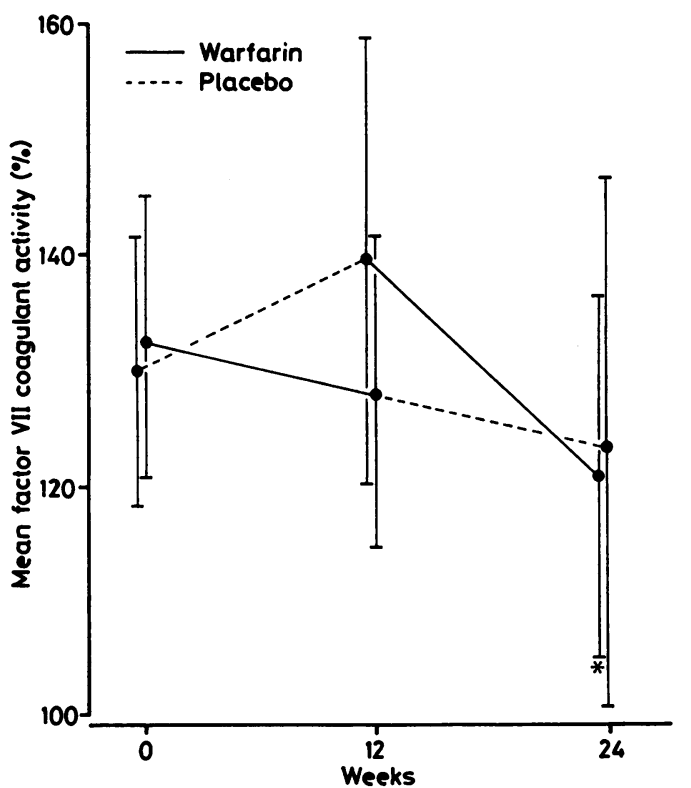

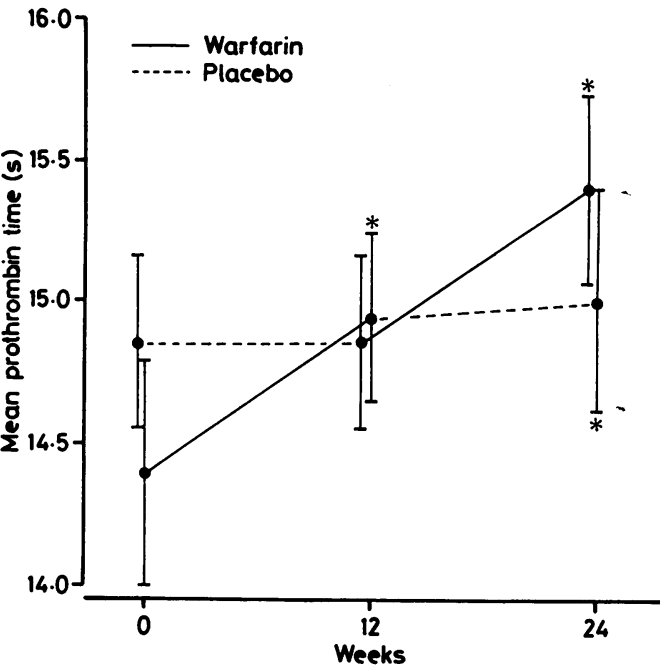

Figure 3 Prothrombin times (mean (2SE)) during treatment with a fixed daily dose of $1 \mathrm{mg}$ warfarin and during treatment with placebo. * Significant difference compared with baseline, $p<0.05$.

VII coagulant activity in the two order groups. This showed that the fall in factor VII coagulant activity in the men receiving warfarin first did not achieve statistical significance but in those receiving warfarin second the reduction in factor VII coagulant activity was significant $(p=0.05)$. There was a nonsignificant increase in factor VII coagulant activity in those receiving placebo first and a non-significant reduction in those receiving placebo after warfarin.

There was a significant difference at baseline $(\mathrm{p}<0.01)$ in the prothrombin time between those receiving warfarin first and placebo first, which has been allowed for in the analysis (fig 3). On no occasion was the prothrombin time prolonged outside the normal range (13.5-17.5 s) but both groups showed that the prothrombin time during warfarin treatment was significantly longer than the immediate pretreatment values $(p<0.05)$. In those receiving warfarin first the prolongation of the prothrombin time was maintained at the end of the placebo period. No clinical side effects of treatment were reported.

\section{Discussion}

The study showed that a very small fixed dose of warfarin ( $1 \mathrm{mg}$ daily) significantly reduced factor VII coagulant activity in healthy male Post Office workers with high activities. The fixed daily dose of $1 \mathrm{mg}$ of warfarin that we used is much less than the range of mean adjusted maintenance daily doses (2.45-8.76 $\mathrm{mg}$ ) reported in an international survey. ${ }^{9}$ It is also considerably less than the mean daily doses of 5.8 and $4.9 \mathrm{mg}$ used by Hull and colleagues to prevent recurrent venous thrombosis. ${ }^{10}$ In an earlier study we found that the fixed daily dose of $1 \mathrm{mg}$ warfarin significantly reduced the incidence of deep vein thrombosis after major surgery. ${ }^{4}$ This suggested that a small reduction in factor VII coagulated activity achieved with this regimen is clinically effective, and might also protect against the subsequent development of ischaemic heart disease. The effects of 
fixed minidose warfarin have not previously been investigated in individuals predisposed to arterial disease.

The present results suggest that the fixed dose of $1 \mathrm{mg}$ warfarin daily might have beneficial effects on this major risk factor for ischaemic heart disease without disturbance of normal haemostasis. The reduction in factor VII coagulant activity was associated with a parallel prolongation of the prothrombin time, but within the limits of the normal range. The effect on the prothrombin time was more uniform than that on factor VII coagulant activity in the two groups presumably because the prothrombin time unlike the assay for factor VII coagulant activity also reflects depression of additional clotting factors reduced by warfarin-for example factors II and X. The sustained depression of factor VII coagulant activity in the group receiving warfarin first was unexpected and of interest but requires confirmation by further study.

Meade et al showed a positive association between factor VII coagulant activity and the subsequent development of ischaemic heart disease. ${ }^{12}$ This relation seemed even stronger than the well recognised association between raised cholesterol in the subsequent 5 years and the mortality from ischaemic heart disease. Factor VII coagulant activity was high after recent thrombosis ${ }^{11}$ and in several states associated with an increased risk of ischaemic heart disease, including increasing age, ${ }^{56}$ hyperlipidaemia, ${ }^{1213}$ obesity, ${ }^{14}$ high fat intake, ${ }^{15}$ and diabetes. ${ }^{16}$ Raised activity of factor VII coagulant activity was also associated with use of oral contraceptives, ${ }^{17}$ pregnancy, ${ }^{18}{ }^{19}$ and the menopause. ${ }^{20}$

The therapeutic range for the treatment of arterial disease, including myocardial infarction, recommended by the British Society for Haematology is an International Normalised Ratio of between 3.0 and $4.5 .^{21}$ This represents a minimum prolongation of 30 seconds over the upper limit of the normal range with the Manchester Reagent thromboplastin used to measure the prothrombin time in the present study. The maximum prolongation in any of our subjects was $2.25 \mathrm{~s}$ and in no instance did the prothrombin time exceed the upper limit of the normal range.

Oral anticoagulant treatment has been claimed to be effective in secondary prevention of myocardial infarction when given in traditional adjusted doses ${ }^{22}$ but no studies have yet been published on the value of oral anticoagulants in the primary prevention of ischaemic heart disease. A large clinical trial is, however, in progress in the United Kingdom to see whether less intense anticoagulation (target International Normalised Ratio 1.5) with an adjusted dose of warfarin is effective. ${ }^{23}$

The epidemiological evidence indicates that raised activity of factor VII is an important risk factor for ischaemic heart disease. It is possible therefore that reduction of high activities by fixed dose warfarin might be beneficial. To investigate this requires a much larger series of high risk subjects with raised factor VII coagulant activity followed for a considerably longer period. Minidose warfarin may have the advantage of not requiring frequent or regular laboratory monitoring, which is an essential prerequisite and limitation of adjusted dosage.

We thank Mrs $M$ Berry and Mrs $M$ Jarvis, Senior Nursing Advisors to the Post Office (North West) for their valuable support; Mr B Farragher, Department of Medical Statistics, Support; Mr B Farragher, Department of Medical Statistics, placebo tablets; and the Pharmacy Department, Withington placebo tablets; and the Phar
Hospital for their cooperation.

1 Meade TW, North WRS, Chakrabarti R, et al. Haemostatic function and cardiovascular death: early results of prospective study. Lancet $1980 ; \mathbf{i}: 1050-4$.

2 Meade TW, Mellows S, Brozovic M, et al. Haemostatic function and ischaemic heart disease: principal results of the Northwick Park Heart Study. Lancet 1986;i.533-7.

3 Bern MM Bothe 1 ern MM, Bothe A Jr, Bistrian B, Champagne CD, Keane MS, Blackburn GL. Prophylaxis against central vein
thrombosis with low-dose warfarin. Surgery 1986;99: thrombosis.

4 Poller L, McKernan A, Thomson JM, Elstein M, Hirsch PJ, Jones JB. Fixed minidose warfarin: a new approach to prophylaxis against venous thrombosis after major surgery. Br Med J 1987;295:1309-12.

5 Brozovic M, Stirling Y, Harricks C, North WRS, Meade TW. Factor VII in an industrial population. $B r J$ Haematol 1974;28:381-91.

6 Balleisen L, Bailey J, Epping P-H, Schulte H, van de Loo J. Epidemiological study on factor VII, factor VIII and fibrinogen in an industrial population: 1 . Baseline data on the relation to age, gender, body-weight, smoking, alcohol, pill-using and menopause. Thromb Haemost 1985;54:475-9.

7 Poller L, Thomson JM, Sear CHJ, Thomas W. Identification of a congenital defect of factor VII in a colony of beagle dogs: the clinical use of the plasma. J Clin Pathol beagle dogs: the

8 Winer BJ. Statistical principles in experimental design. 2nd ed. New York: McGraw-Hill, 1971:149-305.

9 Poller L, Taberner DA. Dosage and control of oral anticoagulants: an international collaborative survey. $\mathrm{BrJ}$ Haematol 1982;51:479-85.

10 Hull R, Hirsh J, Jay R, et al. Different intensities of oral anticoagulant therapy in the treatment of proximal vein thrombosis. N Engl J Med 1982;307:1676-81.

11 Poller L. Thrombosis and factor VII activity. J Clin Pathol 1957;10:348-50.

12 Constantino M, Merskey C, Kudzma DJ, Zucker MB. Increased activity of vitamin K-dependent clotting factors in human hyperlipoproteinaemia-association with
cholesterol and triglyceride levels. Thromb Haemost 1977;38:465-74.

13 Miller GJ, Walter SJ, Stirling Y, Thompson SG, Esnouf MP, Meade TW. Assay of factor VII activity by two techniques: evidence for increased conversion of VII to $\alpha \mathrm{VIIa}$ in hyperlipidaemia, with possible implications for ischaemic heart disease. Br J Haematol 1985;59:249-58.

14 Meade TW. Epidemiology of atheroma, thrombosis and ischaemic heart disease. In: Bloom AL, Thomas DP, eds. Haemostasis and thrombosis. 2nd ed. Edinburgh: Churchill Livingstone, 1987:697-720.

15 Miller GJ, Martin JC, Webster J, et al. Association between dietary fat intake and plasma factor VII coagulant activity -a predictor of cardiovascular mortality. Atherosclerosis 1986;60:269-77.

16 Fuller JH, Keen H, Jarrett RJ, et al. Haemostatic variables associated with diabetes and its complications. Br Med J 1979;ii:964-6.

17 Thomson JM, Poller L. Oral contraceptive hormones and blood coagulability. Br Med J 1965;ii:270-3.

18 Alexander B, Meyers L, Kenny J, Goldstein R, Gurewich V, Grinspoon $\mathrm{L}$. Blood coagulation in pregnancy: proconvertin and prothrombin, and the hypercoagulable state. $N$ vertin and prothrombin, and the
Engl J Med 1956;254:358-63.

19 Stirling Y, Woolf L, North WRS, Seghatchian MJ, Meade TW. Haemostasis in normal pregnancy. Thromb Haemost TW. Haemostasis

20 Meade TW, Haines AP, Imeson JD, Stirling Y, Thompson SG. Menopausal status and haemostatic variables. Lancet 1983;i:22-4.

21 Poller L. Advances in oral anticoagulant treatment. In: Poller $\mathrm{L}$, ed. Recent advances in blood coagulation 4. Edinburgh: Churchill Livingstone, 1985:191-214.

22 Sixty-Plus Reinfarction Study Group. A double-blind trial to assess long-term oral anticoagulant therapy in elderly patients after myocardial infarction. Lancet 1980;ii: 989-94.

23 Meade TW, Wilkes HC, Stirling Y, Brennan PJ, Kelleher C, Browne CW. Randomised controlled trial of low dose warfarin in the primary prevention of ischaemic heart disease in men at high risk: design and pilot study. Eur Heart $J$ 1988;9:836-43. 